\title{
Successful Treatment of Preterm Labor in Association with Acute COVID-19 Infection
}

\author{
Paul C. Browne, MD ${ }^{1}$ Jennifer B. Linfert, $M^{1}$ Emilio Perez-Jorge, $M D^{2}$ \\ ${ }^{1}$ Department of Obstetrics and Gynecology, Lexington Medical \\ Center, West Columbia, South Carolina \\ ${ }^{2}$ Division of Infectious Diseases, Department of Internal Medicine, \\ Lexington Medical Center, West Columbia, South Carolina \\ Address for correspondence Paul C. Browne, MD, Lexington \\ Maternal-Fetal Medicine, Lexington Medical Center, 222 East Medical \\ Lane, Suite 300, West Columbia, SC 29169 \\ (e-mail: pcbrowne@lexhealth.org).
}

Am J Perinatol 2020;37:866-868.
Abstract
Keywords
- COVID-19
- coronavirus
- pregnancy
- pregnancy complication
- preterm labor
- azithromycin

Novel coronavirus disease 2019 (COVID-19) infection occurring during pregnancy is associated with an increased risk of preterm delivery. This case report describes successful treatment of preterm labor during acute COVID-19 infection. Standard treatment for preterm labor may allow patients with acute COVID-19 infection to recover without the need for preterm delivery.

\section{Key Points}

- Acute COVID-19 infection is associated with a high rate of preterm delivery.

- Standard treatment for preterm labor such as intravenous magnesium sulfate, antepartum steroid therapy and antibiotic prophylaxis for group B streptococcus infection were effective in this patient.

- In the absence of maternal or fetal compromise, acute COVID-19 infection is not an indication for early elective delivery.

Preterm labor is associated with many acute viral infections such as influenza and severe acute respiratory syndrome (SARS). ${ }^{1,2}$ Vaccination against influenza has been shown to decrease the risk of preterm birth in pregnant patients who are exposed to influenza. ${ }^{3}$ The novel coronavirus disease 2019 (COVID-19) causes a multiorgan infection and most often manifests clinically as fever, cough, dyspnea, and myalgias. ${ }^{4}$ Emerging case studies suggest a preterm delivery rate as high as $47 \% .^{5}$ The current case report describes a patient who experienced preterm labor in association with acute COVID-19 infection. The preterm labor resolved with standard treatment, avoiding an extremely low birth weight delivery.

received

April 8, 2020

accepted after revision

April 12, 2020

published online

April 24, 2020

\section{Case Report}

The patient was a 33-year-old married, African American female G1 with estimated date of delivery July 7, 2020. The patient's medical history was remarkable for asthma and migraine headaches. The patient's medications included a prn albuterol inhaler and fexofenadine. The patient spontaneously conceived a dichorionic/diamniotic twin gestation. She was treated for hyperemesis gravidarum and acid reflux in the first trimester. The patient attended a church where multiple church members contracted COVID-19 infection. The patient had multiple contacts with COVID-19-positive family members, including two who later died of the infection.

Copyright $\odot 2020$ by Thieme Medical Publishers, Inc., 333 Seventh Avenue, New York, NY 10001, USA. Tel: +1(212) 760-0888.
DOI https://doi.org/ 10.1055/s-0040-1709993. ISSN 0735-1631. 
The patient developed clinical symptoms consistent with COVID-19 at 23 weeks of gestation, including fever, cough, and myalgias. Using an approved screening protocol, she was tested for influenza, group A streptococcus infection, and COVID-19. Her CBC showed an elevated white blood cell (WBC) count of $16,400 / \mathrm{mL}$ with $81 \%$ granulocytes. Her rapid influenza and rapid streptococcus A screening resulted negative 3 hours after her encounter with emergency medicine. Per protocol, the patient was sent home to self-quarantine for 14 days, pending the results of her COVID-19 screening test. She was prescribed azithromycin and a prednisone taper for suspected bronchitis. She was advised to take acetaminophen for fever and to continue her current medications.

The patient returned 9 days after her PCP visit to her OB provider complaining of contractions and increased vaginal discharge. She was still under self-quarantine. Her upper respiratory symptoms were improving and her fever had resolved essentially 7 to 8 days prior. Her COVID-19 screening test was still pending. She was afebrile (temperature, $98.3^{\circ} \mathrm{F}$ ). Clinical cervical examination was closed. Transvaginal ultrasound of the cervix showed funneling to the level of the exocervix.

The patient was admitted to the hospital for tocolysis, antepartum steroid therapy, and group B strep prophylaxis. Admission CBC showed hemoglobin ( $\mathrm{Hg}), 10.7$ g\%; hematocrit (Hct), 31.4\%; WBC, 23,800/mL; and platelets, 252,000/ $\mathrm{mL}$. Admission vital signs showed temperature was $99.2^{\circ} \mathrm{F}$; pulse, $100 \mathrm{bpm}$; respirations, $16 / \mathrm{min}$, blood pressure (BP), 112/75; and $\mathrm{O}_{2}$ saturation, $97 \%$ on room air. Fetal monitoring was reassuring for both twins. There were no episodes of fetal tachycardia noted on the monitor. Uterine contractions were noted which resolved with intravenous (IV) fluid hydration and IV magnesium sulfate tocolysis ( $4 \mathrm{~g}$ IV bolus and $2 \mathrm{~g} / \mathrm{h}$ ). The patient received betamethasone $12 \mathrm{mg}$ intramuscular injection (IM) q 24 hours for 2 days and IV ampicillin $2 \mathrm{~g}$ q 6 hours. Her group B streptococcus screening on admission later returned negative. The patient's initial COVID-19 screen returned positive on hospital day 3, 11 days after the original specimen was collected. A repeat transvaginal ultrasound examination showed stable cervical funneling to the level of the exocervix. The exocervix was dilated $1.5 \mathrm{~cm}$ on ultrasound. The patient's magnesium was discontinued at 07:30 a.m. on hospital day 3 after 39 hours of infusion. The patient's contractions after discontinuing the magnesium did not recur. The patient's COVID-19 screen was repeated on hospital day 3 and remained positive. The patient was discharged home to continue self-quarantine. She was asked to restrict sexual activity, limited physical activity, and hydrate. The patient's COVID-19 tests were repeated 21 and 22 days after her first reported positive test and were negative. The patient remains pregnant at 27 weeks of gestation at the time of this submission.

\section{Discussion}

The potential complications of acute COVID-19 during pregnancy are beginning to emerge in the medical literature. ${ }^{5,6}$ Severe maternal viral infections are associated with an increased risk of preterm labor. ${ }^{5}$ The current case describes preterm labor which occurred during an acute COVID-19 infection in a primigravid with twins. The patient had multiple contacts with known COVID-19-positive family members, including two who died from the infection. The patient had an underlying cardiopulmonary condition, asthma, which may have increased her risk of severe disease. The patient was treated with azithromycin early in the course of her COVID-19 infection. Azithromycin therapy appears to be effective in ameliorating severe viral pneumonia in nonpregnant cases of COVID-19 infection. ${ }^{7}$ The patient also received a prednisone taper early in her infection. Emerging literature also suggests a possible benefit from immunosuppression with hydroxychloroquine or steroids in COVID-19 infection. ${ }^{8}$ The patient did not develop severe pulmonary infection and did not require intensive care (ICU) care or ventilator assistance. She responded well to traditional treatment for preterm labor with magnesium neuroprotection/tocolysis, antepartum steroid therapy, and group B streptococcus prophylaxis.

The delay in COVID-19 testing results (11 days) reinforces the current public health guidelines that all patients with symptoms suggestive of COVID-19 infections should be treated as presumptive positive cases until their testing returns negative. The patient's episode of preterm labor occurred during her period of self-quarantine, approximately 9 days after presenting with symptoms of an upper respiratory infection. The patient was treated empirically with azithromycin and prednisone early in her clinical course, which may have had a beneficial effect on her overall outcome.

The current case highlights that standard treatment for preterm labor appears to be effective during an acute COVID19 infection. Acute COVID-19 infection should not be an indication for early elective delivery unless maternal or fetal decompensation occurs during treatment. The use of patient isolation and personal protective equipment (PPE) during treatment of patients, with suspected but not proven COVID19 infection, is essential for protecting health care workers who provide care for pregnant women. Vaccination against COVID-19, once available, may reduce the risk of preterm labor in COVID-19 exposed pregnant women.

Conflict of Interest

None declared.

\section{References}

1 Meijer WJ, van Noortwijk AG, Bruinse HW, Wensing AM. Influenza virus infection in pregnancy: a review. Acta Obstet Gynecol Scand 2015;94(08):797-819

2 Theiler RN, Rasmussen SA, Treadwell TA, Jamieson DJ. Emerging and zoonotic infections in women. Infect Dis Clin North Am 2008; 22(04):755-772

3 Nunes MC, Aqil AR, Omer SB, Madhi SA. The effects of influenza vaccination during pregnancy on birth outcomes: a systematic review and meta-analysis. Am J Perinatol 2016;33(11):1104-1114

4 Cao Y, Liu X, Xiong L, Cai K. Imaging and clinical features of patients with 2019 novel coronavirus SARS-CoV-2: A systematic review and meta-analysis. J Med Virol 2020 (e-pub ahead of print). Doi: 10.1002/jmv.25822 
868 Successful Treatment of Preterm Labor of COVID-19-Positive Patient Browne et al.

5 Mullins E, Evans D, Viner RM, O’Brien P, Morris E. Coronavirus in pregnancy and delivery: rapid review. Ultrasound Obstet Gynecol 2020 (e-pub ahead of print). Doi: 10.1002/uog.22014

6 Schwartz DA. An analysis of 38 pregnant women with COVID-19, their newborn infants, and maternal-fetal transmission of SARSCoV-2: maternal Coronavirus infections and pregnancy outcomes. Arch Path Lab Med 2020. Doi: 10.5858/arpa.20200901-SA
7 Centers for Disease Control and Prevention. Information for clinicians on therapeutic options for COVID-19 patients. Available at: https://www.cdc.gov/coronavirus/2019-ncov/hcp/therapeutic-options.html. Accessed April 8, 2020

8 Mehta P, McAuley DF, Brown M, Sanchez E, Tattersall RS, Manson JJ; HLH Across Speciality Collaboration, UK. COVID-19: consider cytokine storm syndromes and immunosuppression. Lancet 2020;395(10229):1033-1034 